OPEN ACCESS

Edited by:

Rufus Johnstone,

University of Cambridge,

United Kingdom

Reviewed by:

Allen J. Moore,

University of Georgia, United States

Stephen T. Trumbo, University of Connecticut,

United States

*Correspondence:

Per T. Smiseth

per.t.smiseth@ed.ac.uk

Specialty section:

This article was submitted to Behavioral and Evolutionary Ecology,

a section of the journal

Frontiers in Ecology and Evolution

Received: 02 May 2019 Accepted: 08 October 2019 Published: 24 October 2019

Citation:

Smiseth PT (2019) Coordination, Cooperation, and Conflict Between Caring Parents in Burying Beetles.

Front. Ecol. Evol. 7:397. doi: 10.3389/fevo.2019.00397

\section{Coordination, Cooperation, and Conflict Between Caring Parents in Burying Beetles}

\author{
Per T. Smiseth* \\ School of Biological Sciences, Institute of Evolutionary Biology, University of Edinburgh, Edinburgh, United Kingdom
}

Much of our current understanding of coordination, cooperation, and conflict between male and female parents caring for their joint offspring derives from studies conducted on birds. However, biparental care is not unique to birds but has evolved repeatedly in a wide range of other taxa, including mammals, reptiles, amphibians, fishes, insects, and crustaceans. Here I highlight how recent studies on burying beetles in the genus Nicrophorus provide new and complementary insights into biparental care to studies conducted on birds. Firstly, coordination between parents might be more complex than traditionally recognized, often involving multiple mechanisms such as negotiation and direct assessment of partner's state. Secondly, coordination is not restricted to parental care, but extends to other interactions between caring parents such as interactions over food consumption from a shared resource. Finally, cooperation may have a stronger impact on coordination between parents than has been traditionally recognized. I suggest that, in order to expand our understanding of coordination, cooperation, and conflict between male and female parents, we now need to extend empirical work to a wider range of taxa, develop new experimental designs for detecting alternative mechanisms of coordination, and use of multiple experimental designs across all taxa.

Keywords: biparental care, Nicrophorus, negotiation, perturbation experiments, sexual conflict

\section{INTRODUCTION}

Biparental care often involves coordination between male and female parents, whereby each parent adjusts its contribution toward care based on its partner's contribution (Harrison et al., 2009; Lessells, 2012). The evolution of such coordination reflects the complex balance between cooperation and conflict that ensues when male and female parents care for their joint offspring. On the one hand, biparental care evolves because the two parents benefit by cooperating to provide care (Maynard Smith, 1977). Yet, on the other hand, the evolution of biparental care gives rise to conflict over how much care each parent should contribute. Such conflict arises because the benefits of care to the offspring depend on the combined contributions by the two parents, whilst the cost of care to each parent is determined by its personal contribution (Lessells, 2012). As a consequence, each parent should be under selection to withhold care when working jointly with a partner, thereby shifting as much of the workload as possible over to its partner (Parker, 1985). Thus, coordination should be based on mechanisms that allow cooperation between male and female parents to be evolutionarily stable despite the destabilizing impact of conflict (Lessells, 2012).

Much of our current understanding of coordination, cooperation, and conflict between male and female parents derives from studies on birds (Harrison et al., 2009; Lessells, 2012). 
This taxonomic bias reflects that biparental care is common in birds, occurring in more than $90 \%$ of bird species (Cockburn, 2006). Furthermore, in birds, it is relatively straightforward to conduct experiments in the wild and monitor the amount of care provided by each of the two parents. Nevertheless, biparental care is not unique to birds but has evolved repeatedly in a wide range of other taxa, including mammals, reptiles, amphibians, fishes, insects, and crustaceans (Balshine, 2012; Trumbo, 2012). Thus, it is important to supplement studies on birds with studies on these non-avian taxa. Such work would help establish the extent to which insights based on studies of birds generalize to other taxa. Perhaps more importantly, some non-avian taxa, such as insects, may allow for alternative designs than those that are available for birds, thereby providing a potential source of novel insights into biparental care. Although biparental care is very rare in insects (Trumbo, 2012; Wong et al., 2013; Smiseth, 2014), it has evolved in a small number of species, including burying beetles of the genus Nicrophorus (Eggert and Müller, 1997). Recently, burying beetles have attracted interest as a non-avian laboratory model system for the study of coordination, cooperation, and conflict in families. Here I review recent work on biparental care in burying beetles and discuss how this work complements work on biparental care in birds.

\section{MECHANISMS OF COORDINATION}

Identifying mechanisms of coordination between the two parents is a key priority in this field as it provides important insights into how cooperation can remain evolutionarily stable in spite of conflict between parents (Lessells, 2012). Work on birds has focused primarily on two mechanisms-negotiation (also known as incomplete compensation) and conditional cooperation (also known as turn-taking or alternation) -in which each parent adjusts its own contribution based on information on its partner's workload (Harrison et al., 2009; Lessells, 2012; Johnstone et al., 2014). Negotiation occurs when the focal parent responds to a reduction in its partner's workload by increasing its own contribution but not such that it fully matches the partner's reduction ("incomplete compensation") (McNamara et al., 1999). Meanwhile, conditional cooperation occurs when the two parents take turns or alternate their contributions toward parental care (Johnstone et al., 2014). There is good evidence for negotiation, deriving mainly from handicapping experiments conducted on several species of birds (Harrison et al., 2009). The rationale of such experiments is to reduce the contribution of one parent by imposing a handicap that increases its cost of care, thereby reducing its workload, and then monitor the response of its partner. Such experiments show that, as predicted, the partner of the handicapped parent tends to increase its contribution toward care but not such that it fully matches the reduction in care by the handicapped parent (i.e., incomplete compensation; Harrison et al., 2009). Evidence for conditional cooperation derives from studies examining the rate of turn-taking or alternation between two parents when provisioning food to their offspring (Johnstone et al., 2014). As discussed in other contributions to this issue, there is debate among avian researchers over the evidence for conditional cooperation (Ihle et al., 2019; Johnstone and Savage, 2019; Santema et al., 2019).

Burying beetles provide a useful complementary system to birds because they allow for alternative experimental designs to those that have been used in the study of biparental care in birds. Burying beetles are similar to birds in that both parents cooperate to provision food and provide other forms care for their joint offspring (Eggert and Müller, 1997), and that offspring beg for food from their parents (Smiseth et al., 2003). However, they differ from birds in that they breed on a fixed resourcethe carcass of a small vertebrate-that has been acquired by the parents prior to breeding (Scott, 1998), and that females tend to provide considerably more care than males (Smiseth and Moore, 2004). Given this sex differences in care, it seems unlikely that conditional cooperation could provide a mechanism for coordination given that turn-taking or alternation necessitates that the two parents have similar workloads. There is however evidence for negotiation based on handicapping experiments and experiments based on random pairing of males and females. Handicapping experiments on two species of burying beetles$N$. orbicollis and N. quadripunctatus-show that male partners increase their contribution toward care males in response to handicapping of the female prior to hatching (Creighton et al., 2015) but not after hatching (Suzuki and Nagano, 2009). Meanwhile, experiments pairing males and females at random to control for potential effects of assortative mating show that there is a negative correlation between the amount of care provided by males and females (Smiseth and Moore, 2004; Mattey and Smiseth, 2015; Pilakouta et al., 2015). Thus, these results show that some of the mechanisms that mediate coordination between parents in birds (i.e., negotiation) also mediate such coordination in burying beetles, confirming that insights from studies on birds generalize at least to some extent to other taxa.

Recent experiments on the burying beetle $N$. vespilloides show that coordination between parents can be more complex than is often recognized in theoretical models of biparental care, involving a combination of negotiation and direct responses to the partner's state. This insight derives from experiments using an experimental design based on the manipulation of a state component of both male and female parents, such as their inbreeding status (Mattey and Smiseth, 2015) or their body size (Pilakouta et al., 2015). The rationale of such designs is to monitor how the state component of the focal parent influences its own contribution toward parental care as well as its partner's contribution. Such designs can be described as perturbation experiments, investigating how the experimental treatment alters the outcome of coordination between the two parents. If our current understanding of the mechanism of coordination between parents is correct, any effect of the manipulation of focal parent's state on its partner contribution should be mediated through the putative mechanism of coordination (e.g., negotiation). These experiments show that each parent adjusts its contribution based on its own state as well as its partner's state (Figure 1A). Furthermore, they show that each parent adjusts its contribution based on its partner's contribution, but that the response to the partner's state is independent of any responses to 
the partner's contribution (Mattey and Smiseth, 2015; Pilakouta et al., 2015). Thus, these experiments show that manipulation of the state of male and female parents alters the outcome of coordination between the two parents, but that this effect cannot be explained by established mechanisms of coordination (e.g., negotiation).

The results from these perturbation experiments have important implications for our understanding of coordination between parents. The reason for this is that negotiation is thought to have evolved as a mechanism whereby each parent obtains information on its partner's ability to provide care (McNamara et al., 1999). This idea assumes that a parent cannot directly assess its partner's parental ability, but that it does so indirectly by monitoring its partner's workload. Thus, recent work on burying beetles suggests that negotiation can play a role as a mechanism of coordination between parents even in situations where parents directly assess components of their partner's state that correlate with their parental ability. It is currently unclear why this is. One potential explanation is that parents obtain different forms of information on their partner's parental ability by monitoring their workload and varying components of their state. For example, monitoring the partner's workload might provide information on its current ability, whilst monitoring their state might provide more liable information on its likely future contributions. An alternative explanation is that coordination is not just about obtaining information on the partner's parental ability but also about coordinating the timing of its own contribution toward care relative to the timing of its partner's contribution. For example, the benefits of care to the offspring may not solely depend on how much care the two parents provide but also on how care is distributed over time. Empirical tests of this idea should carefully consider the sampling protocols used when collecting data on male and female contributions toward parental care. For example, such data may be available from studies using sampling protocols where male and female contributions toward parental care are monitored over substantial parts of the period when parents provision food for their offspring (e.g., Johnstone et al., 2014; Bebbington and Hatchwell, 2016).

\section{COORDINATION OF FOOD CONSUMPTION}

For obvious reasons, prior work on coordination between parents has focused on coordination of the amount of care provided by each parent. However, recent work on burying beetles shows that coordination extends to other aspects of family life, such as food consumption from a shared resource. Burying beetles breed on carcasses of small vertebrates, which serve as a source of food for the larvae as well as the two parents (Boncoraglio and Kilner, 2012). An experiment on $N$. vespilloides shows that there is coordination of food consumption based on a combination of conditional cooperation (i.e., matching) and direct responses to the partner's state (Pilakouta et al., 2016). Females adjust their mass change by matching their partner's mass change, gaining more mass when males gained more mass (Figure 1B). In contrast, males respond directly to their partner's state, gaining more mass when paired to large females that on average consumed
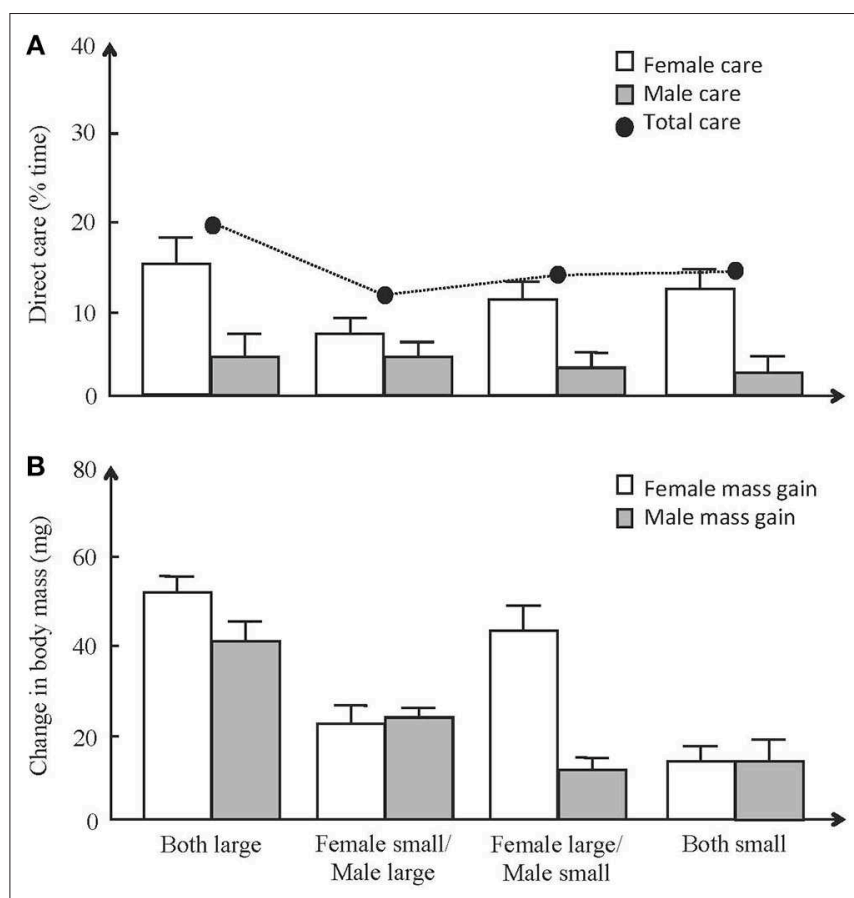

FIGURE 1 | Effects of male and female body size on the amount of care and weight change during breeding of male and female parents in the burying beetle Nicrophorus vespilloides. (A) Mean \pm SE amount of time spent providing direct care by females (open bars) and males (gray bars). The total amount of care offspring received from both parents (filled circles) is shown for each treatment (mean). Parents provided less care when their partner was small (females: $-Z=2.3, p=0.022$; males: $-Z=2.2, p=0.026$ ). (B) Mean \pm SE weight change $(\mathrm{mg})$ over the reproductive attempt for small and large males (black bars) and small and large females (gray bars). Males gained more mass when mated to a large female $(t=-3.2, p=0.002)$, whilst females gained more mass when partner gained more mass $(t=-3.2, p=0.047)$. Used with permission from John Wiley and Sons (A) and Elsevier (B).

more carrion than small females (Figure 1B). This study shows that coordination over food consumption is based on the same mechanisms as those involved in behavioral coordination over the amount of care provided by each parent. There is now a need for studies examining whether these two conflicts are related. For example, if a parent is providing a disproportionate amount of care, its partner may be more tolerant of that parent feeding more from the resource (Pilakouta et al., 2016).

\section{COOPERATION AND CONFLICT}

As stated above, biparental care involves a complex balance between cooperation and conflict between the two parents. Prior work on coordination has emphasized its role as a mechanisms that help maintain the evolutionary stability of biparental care. This emphasis is based on theoretical models predicting that parents should withhold care when working with a partner (Parker, 1985). This model predicts that sexual conflict should have detrimental effects on offspring fitness as offspring receive less care as a consequence of parents withholding care when working with a partner (Parker, 1985). There is some empirical 


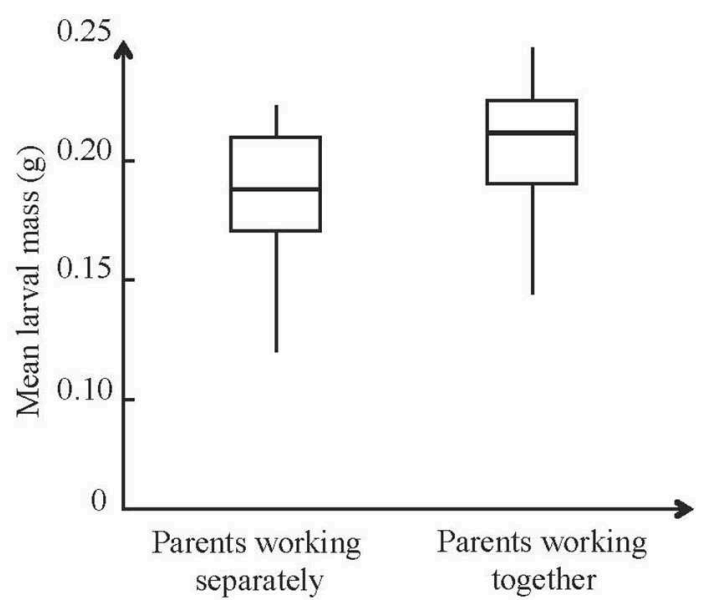

FIGURE 2 | Effects male and female parents working separately or together on larval fitness. Larvae reared by parents working together were heavier at the time of dispersal from the carcass than larvae reared by parents working separately in the burying beetle Nicrophorus vespilloides $\left(\operatorname{LR} \chi_{1,86}^{2}=11: 18, p\right.$ $<0.001)$. Box plot where the thick central line denotes the median, the box the 25th and 75th percentile, and the whiskers denote the 10th and 90th percentiles. Used with permission from Royal Society.

support for this prediction from an experiment on zebra finches, in which the workload per parent was kept constant (Royle et al., 2002). In this species, females withhold care when breeding together with a partner as opposed to when breeding alone, allowing females to save resources for investing in a subsequent breeding attempt. As predicted, sexual conflict has negative consequences for offspring fitness as male offspring reared by females breeding together with a male partner were less attractive to females as adults than males reared by females breeding on their own.

A more recent study on the burying beetle $N$. vespilloides suggests that the effects of cooperation may outweigh those of conflict, and that offspring are better off when the two parents work together (Pilakouta et al., 2018). This study compared levels of parental care when males and females bred together and when males and females bred separately, keeping the amount of resources (i.e., carcass mass) and brood size constant across the two treatments. The study finds no evidence that parents withhold care when working jointly with a partner, and offspring fare better when the two parents work together (Figure 2). These results do not imply that there are no impacts of conflict, only that beneficial effects of cooperation outweigh the detrimental impact of conflict. This finding has important implications because it accounts for the otherwise paradoxical situation where males and females breed jointly even though they would fare better if they bred separately. Furthermore, it suggests that cooperation could have a stronger impact on coordination between parents than traditionally recognized. If so, coordination may be less about resolving conflict as highlighted hitherto, and perhaps be more about enhancing the efficiency of cooperation.
The finding that offspring fare better when the two parents work together (Pilakouta et al., 2018) contrasts with prior work on the burying beetle $N$. vespilloides reporting no difference in reproductive success when the two parents breed together and when they breed on their own (Smiseth et al., 2005). This contrast is likely to reflect differences in experimental design. For example, Pilakouta et al. (2018) removed males at the time they deserted the brood, thereby minimizing the risk that males cannibalize some of their larvae. In contrast, Smiseth et al. (2005) left males with the brood until the larvae dispersed from the brood, in which case the beneficial effects of cooperation may be negated by the detrimental effect of cannibalism by deserting males.

\section{CONCLUDING REMARKS AND PERSPECTIVES}

Here I have highlighted how recent work on burying beetles provides new and exciting insights into coordination, cooperation, and conflict between caring parents. This work highlights that at least some of the insights from studies on birds generalize to other taxa. However, the availability of alternative experimental designs to those used in studies on birds show that coordination of care between the two parents can be more complex than is currently recognized, often involving multiple mechanisms. There is good evidence for some of these mechanisms, such as negotiation, from prior experiments on birds (Harrison et al., 2009) and burying beetles (Smiseth and Moore, 2004), whilst recent experiments on burying beetles provide evidence for other mechanisms based on direct assessment of partner's state (Mattey and Smiseth, 2015; Pilakouta et al., 2015). Furthermore, coordination between parents is not restricted to coordination of how much care each parent should provide, but extends other activities such as the amount of food that each parent consumes from a shared resource. Finally, cooperation may have a stronger impact on coordination between parents than has been traditionally recognized.

What directions should be taken in future work in this field? In my mind, the key question now is whether the contrast between birds, where current evidence suggests that coordination occurs through negotiation (Harrison et al., 2009), and burying beetles, where coordination occurs through a combination of negotiation, conditional cooperation, and direct assessment of partner's state, is real or whether it reflects differences in available designs? In other words, is negotiation the primary mechanism of coordination in birds? Or is this evidence somehow biased, reflecting that handicapping experiments has been the primary tool for studying coordination in birds? Handicapping experiments may provide a biased understanding of coordination if the only mechanism they can detect is negotiation. In contrast, perturbation experiments based on the manipulation of the state of the two parents allow for greater plurality in the mechanisms of coordination. Nevertheless, there are important limitations to such designs, as the mechanisms 
of coordination are often unclear. I suggest that, in order to expand our understanding of coordination, cooperation, and conflict between male and female parents, we now need to extend empirical work to a wider range of taxa, develop new experimental designs for detecting alternative mechanisms of coordination, and use of multiple experimental designs across all taxa.

\section{REFERENCES}

Balshine, S. (2012). "Patterns of parental care in vertebrates" in The Evolution of Parental Care, eds N. J. Royle, P. T. Smiseth, and M. Kölliker (Oxford: Oxford University Press), 62-80.

Bebbington, K., and Hatchwell, B. J. (2016). Coordinated parental provisioning is related to feeding rate and reproductive success in a songbird. Behav. Ecol. 27, 652-659. doi: 10.1093/beheco/arv198

Boncoraglio, G., and Kilner, R. M. (2012). Female burying beetles benefit from male desertion: sexual conflict and counter-adaptation over parental investment. PLoS ONE 7:e31713. doi: 10.1371/journal.pone.0031713

Cockburn, A. (2006). Prevalence of different modes of parental care in birds. Proc. R. Soc. Lond. B 273, 1375-1383. doi: 10.1098/rspb.2005.3458

Creighton, J. C., Smith, A. N., Komendat, A., and Belk, M. C. (2015). Dynamics of biparental care in a burying beetle: experimental handicapping results in partner compensation. Behav. Ecol. Sociobiol. 69, 265-271. doi: $10.1007 / \mathrm{s} 00265-014-1839-\mathrm{z}$

Eggert, A.-K., and Müller, J. K. (1997). "Biparental care and social evolution in burying beetles: lessons from the larder" in The Evolution of Social Behavior in Insects and Arachnids, eds J. C. Choe and B. J. Crespi (Cambridge: Cambridge University Press), 216-236.

Harrison, F., Barta, Z., Cuthill, I. C., and Székely, T. (2009). How is sexual conflict over parental care resolved? A meta-analysis. J. Evol. Biol. 22, 1800-1812. doi: $10.1111 / j .1420-9101.2009 .01792 . x$

Ihle, M., Pick, J. L., Winney., I. S., Nakagawa, S., and Burke, T. (2019). Measuring up to reality: null models and analysis simulations to study parental coordination over provisioning offspring. Front. Ecol. Evol. 7:142. doi: $10.3389 /$ fevo.2019.00142

Johnstone, R. A., Manica, A., Fayet, A. L., Caswell Stoddard, M., RodriguezGironés, M. A., and Hinde, C. A. (2014). Reciprocity and conditional cooperation between great tit parents. Behav. Ecol. 25, 216-222. doi: 10.1093/beheco/art109

Johnstone, R. A., and Savage, J. L. (2019). Conditional cooperation and turn-taking in parental care. Front. Ecol. Evol. 7:335. doi: 10.3389/fevo.2019.00335

Lessells, C. M. (2012). "Sexual conflict" in The Evolution of Parental Care, eds N. J. Royle, P. T. Smiseth, and M. Kölliker (Oxford: Oxford University Press), 150-170.

Mattey, S. N., and Smiseth, P. T. (2015). Complex effects of inbreeding on biparental cooperation. Am. Nat. 185, 1-12. doi: 10.1086/679067

Maynard Smith, J. (1977). Parental investment: a prospective analysis. Anim. Behav. 25, 1-9. doi: 10.1016/0003-3472(77)90062-8

McNamara, J. M., Gasson, C. E., and Houston, A. I. (1999). Incorporating rules for responding into evolutionary games. Nature 401, 368-371. doi: 10.1038/43869

Parker, G. A. (1985). Models of parent-offspring conflict. V. Effects of the behaviour of the two parents. Anim. Behav. 33, 519-533. doi: 10.1016/S0003-3472(85)80075-0

Pilakouta, N., Hanlon, B., and Smiseth, P. T. (2018). Biparental care is more than the sum of its parts: experimental evidence for synergistic effects on offspring fitness. Proc. R. Soc. Lond. B 285:20180875. doi: 10.1098/rspb.2018.0875

\section{AUTHOR CONTRIBUTIONS}

PS reviewed literature and wrote the manuscript.

\section{FUNDING}

PS received financial support from the University of Edinburgh.

Pilakouta, N., Richardson, J., and Smiseth, P. T. (2015). State-dependent cooperation in burying beetles: parents adjust their contribution towards care based on both their own and their partner's size. J. Evol. Biol. 28, 1965-1974. doi: $10.1111 /$ jeb. 12712

Pilakouta, N., Richardson, J., and Smiseth, P. T. (2016). If you eat, I eat: resolution of sexual conflict over consumption from a shared resource. Anim. Behav. 111, 175-180. doi: 10.1016/j.anbehav.2015.10.016

Royle, N. J., Hartley, I. R., and Parker, G. A. (2002). Sexual conflict reduces offspring fitness in zebra finches. Nature 416, 733-736. doi: 10.1038/41 $6733 \mathrm{a}$

Santema, P., Schlicht, E., and Kempenaers, B. (2019). Testing the conditional cooperation model: what can we learn from parents taking turns when feeding offspring? Front. Ecol. Evol. 7:94. doi: 10.3389/fevo.2019. 00094

Scott, M. P. (1998). The ecology and behavior of burying beetles. Ann. Rev. Entomol. 43, 595-618. doi: 10.1146/annurev.ento.43.1.595

Smiseth, P. T. (2014). "Parental care" in The Evolution of Insect Mating Systems, eds D. M. Shuker and L. W. Simmons (Oxford: Oxford University Press), 221-241.

Smiseth, P. T., Darwell, C. T., and Moore, A. J. (2003). Partial begging: an empirical model for the early evolution of offspring signalling. Proc. R. Soc. Lond. B 270, 1773-1777. doi: 10.1098/rspb.2003.2444

Smiseth, P. T., Dawson, C., Varley, E., and Moore, A. J. (2005). How do caring parents respond to mate loss? Differential response by males and females. Anim. Behav. 69, 551-559. doi: 10.1016/j.anbehav.2004.06.004

Smiseth, P. T., and Moore, A. J. (2004). Behavioral dynamics between caring males and females in a beetle with facultative biparental care. Behav. Ecol. 15, 621-628. doi: 10.1093/beheco/arh053

Suzuki, S., and Nagano, M. (2009). To compensate or not? Caring parents respond differentially to mate removal and mate handicapping in the burying beetle, Nicrophorus quadripunctatus. Ethology 115, 1-6. doi: 10.1111/j.1439-0310.2008.01598.x

Trumbo, S. T. (2012). "Patterns of parental care in invertebrates" in The Evolution of Parental Care, eds N. J. Royle, P. T. Smiseth, and M. Kölliker (Oxford: Oxford University Press), 81-100.

Wong, J. W. Y., Meunier, J., and Kölliker, M. (2013). The evolution of parental care in insects: the roles of ecology, life history and the social environment. Ecol. Entomol. 38, 123-137. doi: 10.1111/een.12000

Conflict of Interest: The author declares that the research was conducted in the absence of any commercial or financial relationships that could be construed as a potential conflict of interest.

Copyright (c) 2019 Smiseth. This is an open-access article distributed under the terms of the Creative Commons Attribution License (CC BY). The use, distribution or reproduction in other forums is permitted, provided the original author(s) and the copyright owner(s) are credited and that the original publication in this journal is cited, in accordance with accepted academic practice. No use, distribution or reproduction is permitted which does not comply with these terms. 